\title{
281 Malignant tumours of the oropharynx
}

A Sites include faucial pillars, soft palate, tonsil, base of tongue, vallecula and posterior pharyngeal wall.

B The commonest site is the base of tongue.

C Squamous carcinoma, lymphoma and salivary gland tumours occur in that order of frequency.

D Lymph node metastases are rare in squamous carcinoma.

E Full TNM classification requires a CT scan.

\section{Lymphoma of the oropharynx}

A Most cases are Hodgkin's.

B The B-cell is the commonest cell of origin.

C Burkitt's lymphoma is associated with Herpes simplex virus.

D Investigations should include exploratory laparotomy.

E Complete surgical excision is the treatment of choice.

\section{Treatment of squamous carcinoma of the tonsil}

A Surgery is of no benefit if there are lymph node metastases.

B Radiotherapy is only given for palliation.

C A pectoralis major myocutaneous flap can be used to reconstruct after resection of the primary and radical neck dissection.

D The general condition of the patient is of major prognostic significance.

E Cisplatin has been shown to prolong survival in Phase III trials. 